\title{
Perspectives on cross-diagnostic trajectories and outcomes in children's mental health
}

\author{
Shira Barzilay ${ }^{1} \cdot$ Alan Apter $^{1,2,3}$
}

Published online: 1 July 2020

○) Springer-Verlag GmbH Germany, part of Springer Nature 2020

"We are worried about our 14-year old son Sam. Over the course of six months of treatment, he does no longer self-harm or feel depressed and more often hangs out with his friends, but he is increasingly anxious about schoolwork and will drop out if he keeps avoiding his assignments".

These words of concerned parents highlight some of the most pervasive challenges experienced by children while in mental health treatment. Community mental health services utilize progress and outcome evaluations for these families. In fact, many mental health professionals and treatment clinics are required to measure the success rate of various interventions. This is done for the benefit of the individual patient as well as to contribute evidence informing the value and efficacy of a specific practice or method. However, although there is an abundance of outcome research, the everyday reality of mental health services' evaluation of patients' progress remains extremely complex and multifaceted. Measuring the observed progress and shift in a child's mental health state in the most accurate and least burdensome way can be particularly complicated. Several issues are pertinent to this endeavor. Firstly, there is often a non-linear change over time across psychiatric domains, and a child may transition from one domain of psychopathology to another. Second, some crucial aspects of child's mental health and wellbeing, such as familial and social relationships as well as school and leisure activities, are not reflected in psychiatric symptom checklists. In fact, these factors are often left to be marginally assessed and reported as footnotes to psychiatric diagnoses by general estimations. Lastly, improvement is in the eye of the beholder. That is, in every child's mental health

Alan Apter

eapter@clalit.org.il

1 Fienberg Child Study Center, Schneider Children' Medical Center of Israel, Tel Aviv University, Tel Aviv, Israel

2 Interdisciplinary Center Herzliya, Herzliya, Israel

3 Ruppin Academic Center, Emek Hefer, Israel case, there are at least three different perspectives: that of the child, the parent(s), and their teachers. These perspectives are known to be often inconsistent. This month's issue sees the publication of two papers addressing distinct, however, complementary, features of the above challenges: the stability of psychopathological symptoms vs. transition [1] and the psychosocial functioning of the individual beyond psychopathology [6]. These are reviewed within the context of recent approaches to psychopathology and trans-diagnostic trajectories in child's mental health.

\section{Transition over time in psychopathology}

Comorbidity of psychiatric disorders is often the rule, rather than the exception. Furthermore, it is important that children's psychiatric symptoms be viewed in the context of their specific developmental pathways. To best determine a child's prognosis and construct a viable treatment plan, it is vital to understand various diagnostic trajectories. In other words, it is paramount to discern the stability of a diagnosis and previse how it may transition or progress into another set of psychiatric symptoms and difficulties. O'Connor et al. [1] analyzed 12,543 clinical records of child and adolescent mental health services in Britain. Findings revealed that approximately one in five patients had undergone a diagnosis adjustment over time (i.e., revision or supplementation of previously recorded diagnoses at least 30 days after Index Diagnosis). The most common diagnostic bi-directional longitudinal sequences were within the internalizing (affective and anxiety or stress-related disorders) and externalizing (hyperkinetic and conduct disorders) clusters. However, there was little longitudinal transition overlap across externalizing and internalizing clusters. This large-scale study emphasizes the high prevalence and typical presentation of diagnostic adjustments in children.

Comorbidity of symptoms, however, does not necessarily explain the greater picture involving a multitude of interrelationships between etiologies of various psychopathological 
symptoms. These symptoms can influence one another in several directions, or they may co-exist based on a third underlying factor. The network approach addresses some of these questions by estimating relationships between the vicissitudes of psychiatric symptoms over time. Furthermore, this approach to psychopathology proposes that disorders develop from complex associations between different symptoms and domains. Mapping these associations across psychiatric disorders over time may allow better understanding of the mechanisms and pathways underlying psychiatric comorbidities. McElroy et al. [2] examined the longitudinal network structure of internalizing and externalizing psychopathology in a large sample of children and youth aged 7-14 years. Results indicated various interactions between internalizing and externalizing psychopathology at both the symptomatic and the disorder-level, suggesting mutual influences between internalizing and externalizing psychopathology may occur in childhood through adolescence. This contrasts with the results reported by O'Connor [1]. Interestingly, the most stable interaction at the disorder-level was between depression and oppositional defiant disorder. Generalized anxiety disorder and oppositional defiant disorder were the most central disorders in the networks (i.e., most symptoms are associated with these disorders).

The interaction and transition between symptoms may be explained by an emerging approach that originated in adult psychopathology. This proposes a general latent dimension underlying vulnerability to all psychopathological symptoms, termed the $p$ factor. Recent studies supported this approach in childhood and adolescence. Bianchi et al. [3] performed a latent class analysis (LCA) on the Child Behavior Checklist (CBCL) and found a profile characterized by severe emotional and behavioral dysregulation that is mostly associated with the presence of multiple comorbid diagnoses. Moreover, Miller et al. [4] proposed that this general 'dysregulation profile' can already be recognized in 36-months-old preschool children. These findings, among others, support the notion of emotion regulation as a transdiagnostic factor in the development of internalizing and externalizing psychopathology in children and adolescents.

\section{Psychosocial functioning}

An important part of the discussion in children's mental health progress and outcome is each child's level of psychosocial functioning, especially in the context of transdiagnostic approaches. A disruption in a child's functioning and the presence of difficulties in various psychosocial domains not only co-occur with psychiatric symptoms but may also influence them and vice versa. For example, in a large sample of European adolescents, bi-directional longitudinal effects were demonstrated between bullying victimization and depression as well as complex longitudinal associations between victimization and suicidal ideation/attempts [5]. However, it is challenging to thoroughly assess particular psychosocial domains as each life domain (e.g., family, friends, school, or work) may require a long and complicated sets of instruments. Alternatively, short measures may not be psychometrically sound. The study by Casey et al. [6] addresses this by validating a brief and readily available self-report instrument of child and adolescent general psychosocial functioning. The Child Outcome Rating Scale (CORS), consisting of a four-item visual analogue scale, was found to be reliable and valid within clinic and community samples aged 10-15. Furthermore, it was shown to discriminate between clinical and non-clinical samples. Therefore, the CORS may be a feasible as well as robust measure to evaluate important indicators of progress beyond symptom reduction.

\section{Perspectives}

Finally, when mental health professionals and services are requested to gage the progress of a child's mental state, careful attention should be given to the advantages and disadvantages of using different reports. The significance of multiple informants in measuring a child's mental health is well established; however, it is only useful if it provides information about which, if any, of the informants is good. A recent Dutch study [7] validated the Strengths and Difficulties Questionnaire (SDQ) against professional psychiatric diagnoses. As in previous studies, there was limited agreement between adolescents and their parents. The results indicated better predictive accuracy for the SDQ hyperactivity subscale for attention deficit/hyperactivity disorders when reported by parents. The accuracy was improved by including both parent and adolescent reports. The Conduct subscale predicted oppositional defiant and conduct disorders, with the parent as the informant, and the emotional subscale predicted mood and anxiety disorders, with the adolescent as the informant. These findings may help to inform clinicians about the value of each report.

\section{Conclusions}

The assessment of observed progress and change used in children's mental health interventions may benefit from considering longitudinal developmental trajectories, transitions across psychopathological symptoms and transdiagnostic factors, including emotion regulation and psychosocial functioning. Furthermore, interventions may be evaluated by not only symptom reduction, but also by their ability to prevent transitions from one problem area to another [8]. 


\section{References}

1. O'Connor C, Downs J, Shetty H, McNicholas F (2019) Diagnostic trajectories in child and adolescent mental health services: exploring the prevalence and patterns of diagnostic adjustments in an electronic mental health case register. Eur Child Adolesc Psychiatr, pp. 1-13.

2. McElroy E, Shevlin M, Murphy J, McBride O (2018) Co-occurring internalizing and externalizing psychopathology in childhood and adolescence: a network approach. Eur Child Adolesc Psychiatr 27:1449-1457

3. Bianchi V, Brambilla P, Garzitto M, Colombo P, Fornasari L, Bellina M, Bonivento C, Tesei A, Piccin S, Conte S, Perna G, Frigerio A, Castiglioni I, Fabbro F, Molteni M, Nobile M (2017) Latent classes of emotional and behavioural problems in epidemiological and referred samples and their relations to DSM-IV diagnoses. Eur Child Adolesc Psychiatr 26(5):549-557

4. Miller M, Iosif A-M, Young GS, Bell LJ, Schwichtenberg AJ, Hutman T, Ozonoff S (2019) The dysregulation profile in preschoolers with and without a family history of autism spectrum disorder. J Child Psychol Psychiatr 60:516-523
5. Brunstein Klomek A, Barzilay S, Apter A, Carli V, Hoven CW, Sarchiapone M, Kaess M (2019) Bi-directional longitudinal associations between different types of bullying victimization, suicide ideation/attempts, and depression among a large sample of European adolescents. J Child Psychol Psychiatr 60(2):209-215

6. Casey P, Patalay P, Deighton J, Miller SD, Wolpert M (2019) The child outcome rating scale: validating a four-item measure of psychosocial functioning in community and clinic samples of children aged 10-15. Eur Child Adolesc Psychiatr, pp. 1-14.

7. Vugteveen J, De Bildt A, Hartman CA, Timmerman ME (2018) Using the Dutch multi-informant Strengths and Difficulties Questionnaire (SDQ) to predict adolescent psychiatric diagnoses. Eur Child Adolesc Psychiatr 27:1347-1359

8. Barzilay S, Apter A, Snir A, Carli V, Hoven CW, Sarchiapone M, Kaess M (2019) A longitudinal examination of the interpersonal theory of suicide and effects of school-based suicide prevention interventions in a multinational study of adolescents. J Child Psychol Psychiatr 60(10):1104-1111 